\title{
LOCAL CIRCUIT SYNAPTIC INTERACTIONS IN HIPPOCAMPAL BRAIN SLICES ${ }^{1}$
}

\author{
W. DOUGLAS KNOWLES AND PHILIP A. SCHWARTZKROIN ${ }^{2}$
}

Department of Neurological Surgery, University of Washington, Seattle, Washington 98195

\begin{abstract}
The hypothesis that recurrent inhibition in the hippocampus is mediated by inhibitory interneurons was tested with simultaneous intracellular recordings from the CA1 region of guinea pig hippocampal slices in vitro. In recordings from 101 pairs of pyramidal cells, no interactions were detected in $87 \%$ of the pairs. In $13 \%$ of the pyramidal cell pairs, spike trains induced in one cell caused inhibitory postsynaptic potentials (IPSPs) in the second cell. No excitatory interactions were detected. In recordings from 43 pairs of cells, where one cell was a pyramidal cell and the other cell was an interneuron, no interactions were detected in $53 \%$ of the pairs. In $30 \%$ of the interneuron-pyramidal cell pairs, spike trains elicited from the interneuron caused IPSPs in the pyramidal cell. In $28 \%$ of the pairs, spike trains elicited from the pyramidal cell caused excitatory postsynaptic potentials (EPSPs) in the interneurons. In $4 \%$ of these pairs, reciprocal interactions were seen, with the pyramidal cell exciting the interneuron and the interneuron inhibiting the pyramidal cell.

These results support the hypothesis that inhibitory interneurons mediate recurrent inhibition in the hippocampus. However, the data also suggest that the interneurons from which these results were recorded were a subset of inhibitory interneurons distinct from the classical basket cell. These interneurons may mediate both feed-forward and recurrent inhibition in the hippocampus.
\end{abstract}

Experimenters have described hippocampal inputs and outputs (see Lopes da Silva and Arnolds, 1978) and have studied intrahippocampal communication between cell groups (Andersen, 1975), but there are few data concerning local circuit interactions in hippocampus. In particular, although the concept of recurrent inhibition has been quoted widely to account for the powerful inhibition seen in hippocampal pyramidal cells, the existence of inhibitory interneurons in the hippocampus has been inferred only indirectly from the histological (Ramón y Cajal, 1911; Lorente de Nó, 1934; Amaral and Woodward, 1977) and physiological evidence (Andersen, 1975; Andersen et al., 1964a, b; Finch and Babb, 1977; Schwartzkroin and Mathers, 1978). The histologically identified basket cell is the neuron most often associated with the physiologically inferred inhibitory interneuron, but there is little direct evidence to support this association (Schwartzkroin and Mathers, 1978; Turner and Schwartzkroin, 1980). The inhibitory interneuron is postulated to receive excitatory inputs principally from pyramidal cell axon

\footnotetext{
${ }^{1}$ This work was supported by National Science Foundation Grant BNS 79-15115 and National Institutes of Health Grants NS 00413 and NS 04053 from the National Institute of Neurological and Communicative Disorders and Stroke, Public Health Service/Department of Health and Human Services.

${ }^{2}$ Affiliate of the Child Development and Mental Retardation Center, University of Washington.
}

collaterals and to send inhibitory GABAergic processes back to the pyramidal cell somas (Andersen, 1975; Curtis et al., 1970), thus completing a recurrent inhibitory circuit. Due to the relatively small number of basket cells present in the hippocampus, one would expect a great deal of divergence from basket cells to pyramidal cells (Andersen, 1975).

The large size and effectiveness of the inhibitory postsynaptic potentials (IPSPs) seen in pyramidal cells after afferent pathway stimulation suggest that the inhibitory interneurons are important in the functional operation of the hippocampus. For example, it has been hypothesized that loss of inhibitory control is involved in the development of epileptiform activity in the hippocampus (Schwartzkroin and Prince, 1980; Finch and Babb, 1977; Sawa et al., 1963; Dichter and Spencer, 1969) and that some antiepileptic drugs (e.g., diazepam) act by exciting inhibitory interneurons (Lee et al., 1979). Further, it has been postulated that the excitatory effects of enkephalin in hippocampus are due to the inhibition of inhibitory interneurons (Lee et al., 1980; Gähwiler, 1980; Nicoll et al., 1980).

The literature regarding local circuit inhibition in hippocampus has been built up around a hypothesis that has never been verified directly. In this report, we describe the results of simultaneous intracellular recordings from CA1 pyramidal cell pairs and pyramidal cell-interneuron pairs in the in vitro hippocampal slice prepara- 
tion. These experiments show the existence of local inhibitory circuits that can account for recurrent inhibition in hippocampal pyramidal cells.

\section{Materials and Methods}

The in vitro guinea pig hippocampal slice preparation has been described in detail previously (Schwartzkroin, 1975). The major modification made for the present study was that the hippocampus was sliced in the longitudinal direction. This change was made in hopes of preserving more local inhibitory pathways, which are thought to be oriented perpendicularly to the transverse lamellar organization of the excitatory hippocampal circuitry (Struble et al., 1978). After dissection of the hippocampus, both ends were cut away, leaving approximately the middle third. This portion of the hippocampus was cut into 400 - to $450-\mu \mathrm{m}$-thick longitudinal slices oriented in the dorsoventral plane. In two to four of the slices obtained from each hippocampus, the CA1 pyramidal cell layer was clearly visible (usually along with two layers of dentate granule cells). These slices were chosen for recording.

Recording electrodes with intraluminal glass fibers were pulled using a Brown-Flaming puller (Brown and Flaming, 1977) and filled with $4 \mathrm{M}$ potassium acetate and $0.01 \mathrm{M}$ potassium chloride; electrode DC resistances were between 50 and 100 megohms. Electrode signals were amplified by high input impedance DC amplifiers with bridge circuits; constant current pulses could be injected through the recording electrodes for stimulating or polarizing each cell. Extracellular stimulation was carried out with a bipolar electrode formed from etched tungsten wires. Constant current stimuli $(0.1$ msec duration, 0.1 to $1.0 \mathrm{~mA}$ amplitude) were delivered at $0.3 \mathrm{~Hz}$ to the stratum radiatum or the alveus to determine a cell's response to orthodromic and antidromic inputs. Records were stored on magnetic tape and played back later for analysis and photography.

Electrode penetrations were made into, or within 50 $\mu \mathrm{m}$ of, the stratum pyramidale. An impaled neuron was classified as a pyramidal cell or interneuron using physiological criteria described by Schwartzkroin and Mathers (1978). Hyperpolarizing "holding" current (approximately $0.5 \mathrm{nA}$ ) often was applied to the first neuron while searching for a second neuron (within a radius of approximately $150 \mu \mathrm{m}$ of the first). Upon successful penetration of a second cell, it was characterized similarly. Depolarizing pulses ( $100 \mathrm{msec}$ duration, $0.5 \mathrm{nA}$ amplitude, $0.3 \mathrm{~Hz}$ frequency) were injected into one cell to elicit trains of action potentials. The other cell was examined for changes in membrane potential caused by the spike train in the first cell. This procedure then was reversed to test for synaptic interactions in the opposite direction.

\section{Results}

Pyramidal cell-pyramidal cell pairs. The pyramidal cell is by far the most prevalent cell type in the stratum pyramidale of the CA1 region. Characteristics of pyramidal cells have been described in detail (Schwartzkroin, 1975). For purposes of the present study, pyramidal cells were identified by their relatively long duration action potential (1.4 to $2.0 \mathrm{msec}$ ), depolarizing after-potentials, low spontaneous firing rates, discharge of only one or two action potentials in response to stimulation of an orthodromic pathway, and/or antidromic driving from alveus stimulation.

In $87 \%$ of the $101 \mathrm{CA} 1$ pyramidal cell pairs, no interactions were observed between neurons. In $13 \%$ of the pairs, a spike train induced in one pyramidal cell caused an IPSP-like hyperpolarization in the second pyramidal cell. The observed effect occurred in only one direction and was always inhibitory; no excitatory or reciprocal interactions were detected. Figure 1 shows an example of a recording from a synaptically coupled pair of pyramidal cells. In such interactive cell pairs, the hyperpolarization was generally slow and smooth, 2 to $4 \mathrm{mV}$ in amplitude, and continued beyond the duration of the spike train in the presynaptic cell. With injections of $\mathrm{DC}$ depolarizing current $(0.5$ to $1.0 \mathrm{nA})$ into the postsynaptic cell, the amplitude of the postsynaptic hyperpolarization (induced by presynaptic stimulation) increased. With injection of DC hyperpolarizing current $(0.5$ to $1.0 \mathrm{nA})$, the postsynaptic hyperpolarization decreased in amplitude.

Hyperpolarizing pulses injected into one of the cells in pairs of clearly separate neurons never produced observable effects in the other cell. This test was made in all pairs obtained in order to study possible electrical coupling in these cells. In several special instances, however, an effect of hyperpolarizing current pulses passed by one electrode was noted in the recording from the other electrode. The data suggest that, in these cases, the same pyramidal cell was penetrated by both electrodes. Figure 2 shows an example of such recordings. Under these conditions, the electrodes were nearly isopotential, and every spike was recorded by both electrodes. Current pulses delivered by one electrode were recorded with little diminution at the other electrode. Rarely, small, fast voltage events were recorded by one electrode that were not recorded by the other. Such potentials were interpreted as dendritic spikes, since the electrode recording these events was consistently the one further out into the apical dendrites.

Interneuron-pyramidal cell pairs. In a sample of 360 individual neurons recorded intracellularly in the longi-

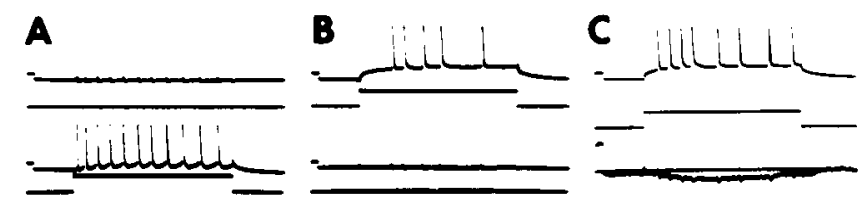

Figure 1. An inhibitory synaptic connection between a pair of pyramidal cells. In this and the following figures, the record from each cell consists of two traces: an intracellular voltage trace (with a $10 \mathrm{mV} \times 5 \mathrm{msec}$ calibration pulse) and a trace showing the current injected intracellularly $(0.5 \mathrm{nA} \times 100 \mathrm{msec}$ for all cells). Spikes have been retouched. $A$, A spike train is elicited in the "postsynaptic" pyramidal cell (lower record) by a current pulse injected into that neuron. $B$, A spike train is similarly elicited in the "presynaptic" pyramidal cell (upper record). $C$, At higher gain, the IPSP produced in the postsynaptic pyramidal cell (lower record) by the presynaptic spike

in (uppe ecord) is apparent (the current trace has been moved to provide a base line). The small, fast peaks in the high gain record are spike artifacts caused by capacity coupling between the electrodes. 


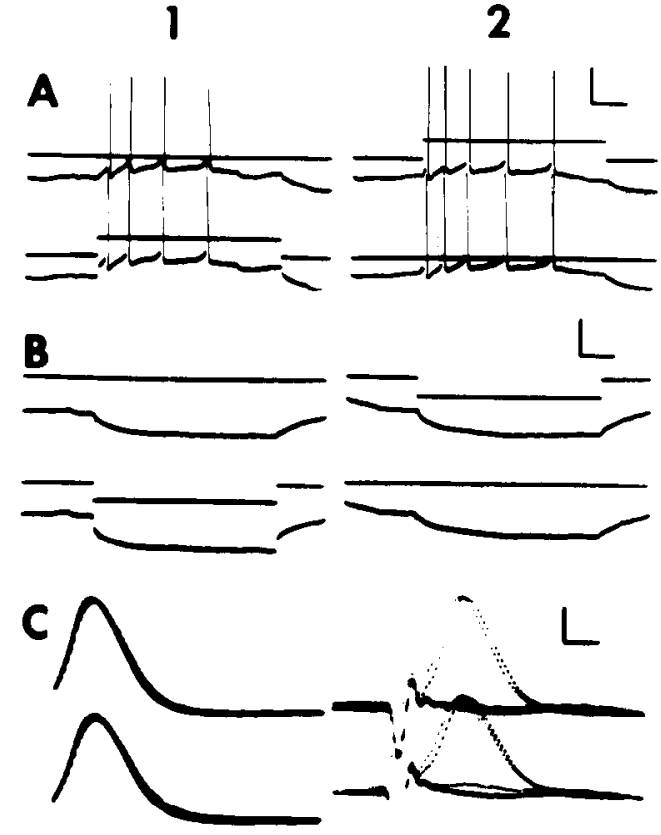

Figure 2. Recordings with two electrodes in the same cell. $A$, Depolarizing pulses injected through one electrode $(A 1)$ and then the other (A2) elicit spike trains recorded equally by both electrodes. $B$, Hyperpolarizing pulses injected through one electrode $(B 1)$ and then the other $(B 2)$ cause nearly identical potentials in both electrodes. $C 1$, Ten superimposed sweeps triggered by spontaneous spikes on the upper record demonstrate the congruence of the spike trains recorded by each electrode. C2, Six superimposed sweeps show that both electrodes recorded nearly identical responses to threshold antidromic extracellular stimulation. Scale bars: $20 \mathrm{mV}$ and 20 msec for $A$ and $B ; 20 \mathrm{mV}$ and $0.5 \mathrm{msec}$ for $C$.

tudinal slices, $5.8 \%$ were identified as interneurons. This percentage is higher than previously reported for the transverse slice $(0.5 \%$ to $2 \%$; Schwartzkroin and Mathers, 1978). Interneurons were easily identified immediately upon penetration. They had brief action potentials (less than $1.2 \mathrm{msec}$ ), prominent after-hyperpolarizations, usually fired spontaneously at high rates (greater than 10 $\mathrm{Hz}$ ), and often had obvious spontaneous synaptic potentials superimposed on the membrane resting potential.

Forty-three interneuron-pyramidal cell pairs were analyzed. Our sample of 43 pairs included 19 different interneurons. With a given interneuron, several pyramidal cells were tested. In $23(53 \%)$ of the interneuronpyramidal cell pairs, no interactions were detected. In 13 $(30 \%)$ of the pairs, induced spike trains in the interneuron (presynaptic cell) caused hyperpolarizations in the pyramidal cell (postsynaptic cell). Figure 3 shows an example of records from an inhibitory interneuron-pyramidal cell pair. The hyperpolarizations were often similar to those seen in the pyramidal cell pairs, being smooth in waveform, 2 to $4 \mathrm{mV}$ in amplitude, and lasting beyond the duration of the presynaptic spike train. These potentials were also enlarged by depolarizing the pyramidal cell and reduced by hyperpolarizing the pyramidal cell.

In $12(28 \%)$ of the interneuron-pyramidal cell pairs, induced spike trains in the pyramidal cell (presynaptic cell) caused excitatory postsynaptic potential (EPSP)like depolarizations, and sometimes action potentials, in the interneuron (postsynaptic cell). Figure 4 illustrates these potentials. These depolarizations recorded in the interneurons were generally larger $(2$ to $10 \mathrm{mV})$ than the hyperpolarizations seen in pyramidal cells when the interneuron was stimulated. The depolarizations were not smooth but rather consisted of individual components elicited by each spike in the pyramidal cell. Thus, the depolarizations appeared to be formed by a summation of individual EPSPs. These depolarizations decreased in size when the interneuron was depolarized and increased when it was hyperpolarized. In those cases where interneuron action potentials were elicited by a presynaptic cell spike, the spike to spike latency was in the range of 2 to $4 \mathrm{msec}$. However, in even the most closely connected cells, only about half of the action potentials in the pyramidal cell elicited interneuron spikes.

One interneuron-pyramidal cell pair was tested for frequency following by injecting 4-msec duration, $0.5-\mathrm{nA}$ amplitude depolarizing pulses at $20 \mathrm{~Hz}$ into the pyramidal cell. Each pulse elicited a spike in the pyramidal cell, but
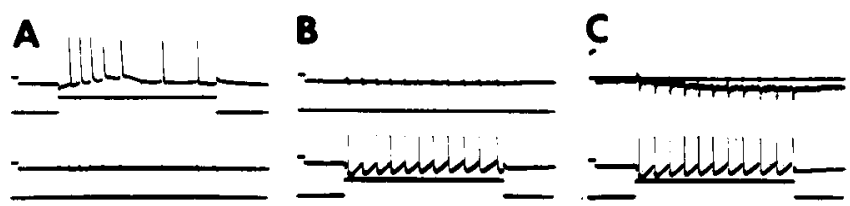

Figure 3. An inhibitory synaptic connection from an interneuron (lower records) to a pyramidal cell (upper records). The same pair of neurons is shown in each panel. $A$, A spike train is elicited in the pyramidal cell by an intracellular current pulse. $B$, A spike train is similarly elicited from the interneuron. $C$, At higher gain, the IPSP produced in the pyramidal cell when the interneuron discharges is apparent (the current trace has been moved to provide a base line).
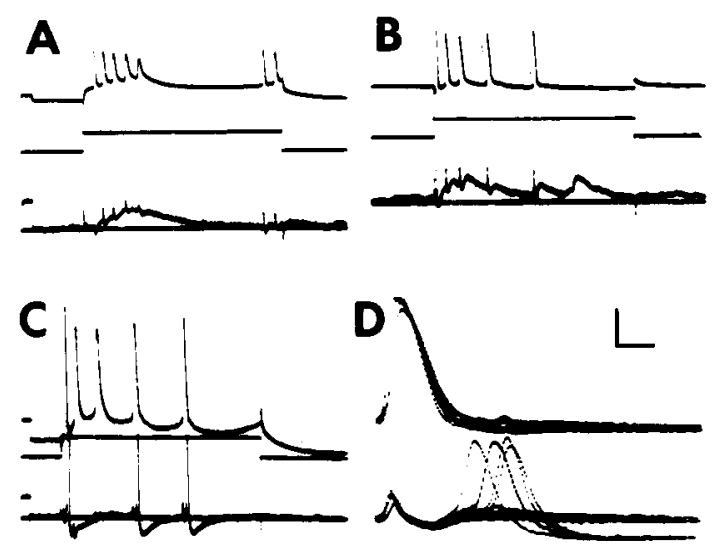

Figure 4. Excitatory synaptic connections from pyramidal cells (upper records) to interneurons (lower records). $A, B$, and $C$, Three different interneuron-pyramidal cell pairs. Depolarizing intracellular current pulses elicit spike trains in the pyramidal cells, producing EPSPs in the interneurons. In $C$, action potentials are sometimes triggered by the interneuron EPSP. The gain of each trace in $B$ is the same as the corresponding trace in $A$. $D$, The same cell pair as is shown in $C$. Ten superimposed sweeps triggered by spontaneous spikes in the pyramidal cell show the variable latency (2 to $3 \mathrm{msec}$ ) and failure rate (six failures) of synaptically driven action potentials in the interneuron. Scale bars for $D$ are $20 \mathrm{mV}$ and $1 \mathrm{msec}$. 
the depolarizations in the interneuron were unable to follow, fading quickly within the first second.

In only two interneuron-pyramidal cell pairs were reciprocal synaptic interactions seen; the pyramidal cell produced depolarizations in the interneuron and the interneuron produced hyperpolarizations in the pyramidal cell (Fig. 5). In the interneuron-pyramidal cell pairs, injection of hyperpolarizing pulses revealed no evidence of electrotonic coupling between interneurons and pyramidal cells.

\section{Discussion}

Both the depolarizing and hyperpolarizing potentials recorded during cellular interactions between hippocampal neurons appeared to be chemically mediated postsynaptic events. The postsynaptic events were dependent on the occurrence of presynaptic action potentials; no effect was seen with presynaptic current injections that were subthreshold for spike initiation. Not every presynaptic action potential caused a postsynaptic potential. Further, the amplitude of the postsynaptic potentials (PSPs) could be varied, as would be expected of PSPs, with manipulation of the postsynaptic cell's resting potential.

Only inhibitory interactions were seen between pairs of pyramidal cells. Latencies from spike firing in a presynaptic pyramidal cell to onset of the IPSP in the postsynaptic pyramidal cell were difficult to determine because of the low amplitude and smooth, gradual onset of the hyperpolarization. Decay of the hyperpolarizations was slow, with a recovery time of 20 to $45 \mathrm{msec}$. If we accept the assumption that the primary pyramidal cell output is excitatory (e.g., Finch and Babb, 1980), any hypothesized monosynaptic inhibitory connections between pyramidal cells would require that a given pyramidal neuron could have both excitatory and inhibitory outputs. To explain inhibitory interactions between py-

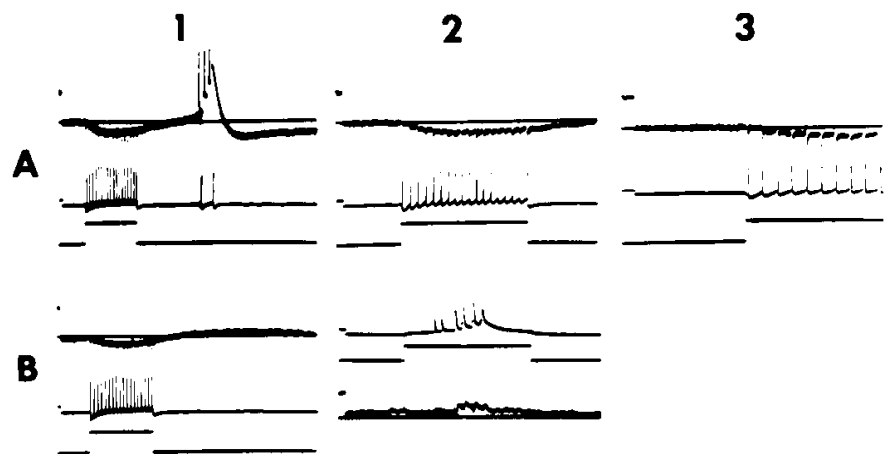

Figure 5. Reciprocal synaptic connections between a pyramidal cell (upper records) and an interneuron (lower records). The same cell pair is shown in each panel. $A$, The same recording is shown at three different sweep speeds. A depolarizing intracellular current pulse elicits a spike train in the interneuron, which produces an IPSP in the pyramidal cell. There is a rebound burst (spikes truncated) in the pyramidal cell $(A 1)$, which produces two synaptically driven spikes in the interneuron. $B 1$, Another pulse-elicited spike train in the interneuron produces an IPSP in the pyramidal cell, but no rebound burst (and no interneuron spikes) occurs. B2, A depolarizing intracellular current pulse elicits action potentials in the pyramidal cell which produce EPSPs in the interneuron (the current trace has been moved to provide a base line). ramidal cells, therefore, it seems best to postulate that at least a disynaptic pathway (involving an intervening interneuron) mediates the IPSP.

The only interaction seen from interneurons to pyramidal cells was inhibitory, and the only interaction from pyramidal cell to interneuron was excitatory. IPSP latency in pyramidal cells induced by interneuron discharge was difficult to determine but can be viewed more easily as due to monosynaptic connections than the pyramidal cell to pyramidal cell inhibition. The reciprocal effect, EPSPs induced in interneurons by pyramidal cell firing, did have a measurable latency consistent with monosynaptic connections. The 2- to 4-msec interspike delay can be accounted for by presynaptic spike transmission along thin, unmyelinated axonal processes, transmitter release, and rise time of postsynaptic EPSPs to trigger postsynaptic spikes.

The neuronal interactions found in the experiments presented here provide evidence which supports the hypothesis (Andersen et al., 1964a) that local circuitry involving inhibitory interneurons mediates recurrent (feedback) inhibition in the hippocampus. Our data also suggest that inhibition is the primary product of local interaction; we saw no evidence of excitatory interneurons, which have been postulated sometimes (Andersen et al., 1969; Licbovitz et al., 1969), nor of excitatory interactions between pyramidal cells. This latter result is different from that of MacVicar and Dudek (1980a) who reported rare excitatory interactions between pyramidal cells. Their recordings, however, were made in the CA3CA4 region of hippocampus where circuitry may differ from that of CA1.

The absence of excitatory local interactions gives little support to the notion that epileptiform activity in hippocampus (at least in the CA1 region) is attributable to recruitment of recurrent excitation. Neither have we found support for the idea that the marked synchrony among neurons in the epileptogenic preparation is due to electrical coupling among the pyramidal cell population. MacVicar and Dudek (1980b) have reported electrical coupling among pyramidal cells in CA3 in a small percentage of pairs; our inability to demonstrate electrical coupling may be attributable to a sampling problem, or again, to a difference in CA1 and CA3 circuitry. The demonstration of fatigue (i.e., EPSP dropout with repetitive stimulation) in the pyramidal cell to interneuron synapse is interesting in light of some theories of epileptogenesis. Finch and Babb (1977) reported a decrement in the response of a presumed interneuron to repetitive extracellular stimulation and interpreted this decrement as synaptic fatigue. They suggested that repetitive stimulation of pyramidal cells may result in fatigue in the recurrent inhibitory pathway, causing loss of inhibitory control and consequent transition to a seizure state. Our observation of fatigue is consistent with this hypothesis.

The significance of our findings of inhibitory local circuitry in hippocampus is dependent, in part, on our ability to identify interneurons using physiologic criteria. The identifications were made on the basis of criteria detailed by Schwartzkroin and Mathers (1978) and reinforced by additional intracellular staining of physiologically identified elements using horseradish peroxidase 
(W. D. Knowles and P. A. Schwartzkroin, unpublished observations). Interestingly, interneurons were encountered more frequently in the longitudinal slices than in transverse slices. This finding may be a result of causing less damage to longitudinally oriented axonal processes of interneurons. Labeled neurons in longitudinal slices were similar in appearance to those identified previously in the transverse slices (Schwartzkroin and Mathers, 1978).

We were surprised that the proportion of pairs in which interneuron activation elicited an IPSP in the pyramidal cell was not higher and that the amplitude of the IPSP (when it was evoked) was not larger. Since extracellular stimulation of orthodromic pathways produces large IPSPs in almost all pyramidal cells, we expected that each interneuron would produce large IPSPs in any pyramidal cell that it contacted and/or that many interneurons would contact each pyramidal cell. Our data did not confirm directly either of our expections but may be consistent with the latter idea. The data further suggest that we recorded from a class of interneurons that comprises a small subset of the inhibitory interneuron pool in hippocampus. A relatively small proportion of pairs showed EPSPs in interneurons when nearby pyramidal cells were stimulated. These results are consistent with the idea that feed-forward inhibition, involving direct excitatory contacts from incoming afferents onto interneurons (Schwartzkroin and Mathers, 1978), plays a role in producing IPSPs in pyramidal neurons. It is possible, of course, that our results were influenced by cellular injury associated with cell penetrations or the in vitro technique. It seems more likely, however, that our data reflect sampling difficulties inherent in intracellular recordings from a large complex population.

In conclusion, we have demonstrated that there is a class of local circuit neurons in the hippocampus which receives excitatory inputs from CA1 pyramidal cells and which sends inhibitory outputs back to the pyramidal cells. These cells are morphologically distinct from pyramidal cells and are easily identified by the waveform of their intracellularly recorded action potential. Since they were located mainly in the stratum pyramidale and since no basket plexuses have yet been seen in intracellular dye injections of these cells, they appear to be a subset of inhibitory interneurons distinct from the classical basket cell. They appear to mediate recurrent as well as feed-forward inhibition in the hippocampus.

\section{References}

Amaral, D. G., and D. J. Woodward (1977) A hippocampal interneuron observed in the inferior region. Brain Res. 124: 225-236.

Andersen, P. (1975) Organization of hippocampal neurons and their interconnections. In The Hippocampus, R. L. Isaacson and K. H. Pribram, eds., Vol. 1, pp. 155-175, Plenum Press, New York.

Andersen, P., J. C. Eccles, and Y. Løyning (1964a) Location of postsynaptic inhibitory synapses on hippocampal pyramids. J. Neurophysiol. 27: 592-607.

Andersen, P., J. C. Eccles, and Y. Løyning (1964b) Pathways of postsynaptic inhibition in the hippocampus. J. Neurophysiol. 27: 608-619.
Andersen, P., G. N. Gross., T. Lømo, and O. Sveen (1969) Participation of inhibitory and excitatory interneurones in the control of hippocampal cortical output. In The Interneuron, M. B. Brazier, ed., pp. 415-432, University of California Press, Berkeley.

Brown, K. T., and D. G. Flaming (1977) New microelectrode techniques for intracellular work in small cells. Neuroscience 2: 813-827.

Curtis, D. R., D. Felix, and H. McLellan, (1970) GABA and hippocampal inhibition. Br. J. Pharmacol. 40: 881-883.

Dichter, M., and W. A. Spencer (1969) Penicillin-induced interictal discharges from the cat hippocampus II. Mechanisms underlying origin and restriction. J. Neurophysiol. 32: 663687.

Finch, D. M., and T. L. Babb (1977) Response decrement in a hippocampal basket cell. Brain Res. 130: 354-359.

Finch, D. M., and T. L. Babb (1980) Neurophysiology of the caudally directed hippocampal efferent system in the rat: Projections to the subicular complex. Brain Res. 197: 11-26.

Gähwiler, B. H. (1980) Excitatory action of opioid peptides and opiates on cultured hippocampal pyramidal cells. Brain Res. 194: 193-203.

Lee, H. K., T. V. Dunwiddie, and B. J. Hoffer (1979) Interaction of diazepam with synaptic transmission in the in vitro rat hippocampus. Naunyn Schmiedeberg's Arch. Pharmacol. 309: 131-136.

Lee, H. K., T. Dunwiddie, and B. Hoffer (1980) Electrophysiological interactions of enkephalins with neuronal circuitry in the rat hippocampus. II. Effects on interneuron excitability. Brain Res. 184: 331-342.

Liebovitz, R., M. Dichter, and W. A. Spencer (1969) Recurrent excitation in hippocampus. Fed. Proc. 28: 455.

Lopes da Silva, F. H., and D. E. A. T. Arnolds (1978) Physiology of the hippocampus and related structures. Annu. Rev. Physiol. $40: 185-216$.

Lorente de Nó, R. (1934) Studies on the structure of the cerebral cortex. II. Continuation of the study on the ammonic system. J. Psychol. Neurol. (Lpz.) 46: 113-117.

MacVicar, B. A., and F. E. Dudek (1980a) Local synaptic circuits in rat hippocampus: Interactions between pyramidal cells. Brain Res. 184: 220-223.

MacVicar, B. A., and F. E. Dudek (1980b) Dye-coupling between CA3 pyramidal cells in slices of rat hippocampus. Brain Res. 196: 494-497.

Nicoll, R. A., B. E. Alger, and C. E. Jahr (1980) Enkephalin blocks inhibitory pathways in the vertebrate CNS. Nature 287: 22-25.

Ramón y Cajal, S. (1911) Histologie du systeme nerveux de l'homme et des vertebres, S. A. Maloine, Paris.

Sawa, M., N. Maruyama, and S. Kaji (1963) Intracellular potential during electrically induced seizures. Electroencephalogr. Clin. Neurophysiol. 15: 209-220.

Schwartzkroin, P. A. (1975) Characteristics of CA1 neurons recorded intracellularly in the hippocampal in vitro slice preparation. Brain Res. 85: 423-436.

Schwartzkroin, P. A., and L. H. Mathers (1978) Physiological and morphological identification of a nonpyramidal hippocampal cell type. Brain Res. 157: 1-10.

Schwartzkroin, P. A., and D. A. Prince (1980) Changes in excitatory and inhibitory synaptic potentials leading to epileptic activity. Brain Res. 183: 61-76.

Struble, R. G., N. L. Desmond, and W. B. Levy (1978) Anatomical evidence for interlamellar inhibition in the fascia dentata. Brain Res. 152: 580-585.

Turner, D. A., and P. A. Schwartzkroin (1980) Steady-state electrotonic analysis of intracellularly stained hippocampal neurons. J. Neurophysiol. 44: 184-199. 